\title{
Optimized Meter Placement in Low Voltage Grids based on Asymmetric State Estimation
}

\author{
Sebastian Hubschneider, Martin Uhrig, Michael Suriyah, Thomas Leibfried \\ Institute IEH \\ Karlsruhe Institute of Technology \\ Karlsruhe, Germany \\ \{sebastian.hubschneider,martin.uhrig,michael.suriyah,thomas.leibfried\}@kit.edu
}

\begin{abstract}
Alongside the ongoing energy system transition towards sustainability new challenges for low voltage grids arise. New technologies connected to those subordinate grids are less predictable, especially decentralized solar plants. Larger loads and a possible reversed power flow lead to increasingly unknown states and can evoke violations of power quality. This paper presents a method to determine an optimized meter placement in low voltage grids using an asymmetric state estimation in order to achieve a cost-efficient monitoring. First, the utilized state estimation method is introduced as well as the usage and parameterization of pseudo measurement values are discussed. Furthermore, a new approach for an optimized meter placement is presented and simulation results for exemplary grids and corresponding power flow data are shown. Subsequent discussions focus on the quality of results subject to the amount as well as the specific positioning of meters placed.
\end{abstract}

Index Terms-Parameter estimation, Power distribution, Power system measurements, Smart grids, State estimation

\section{INTRODUCTION}

Due to technological progress as well as an increasing awareness of sustainability goals and thus renewable power generation, low voltage (LV) grids are subject to change. The increasing amount of decentralized solar plants in conjunction with larger loads and time differences between production and consumption lead to new challenges. The modified load situation of LV grids, which increasingly face reversed loadflow and/or line overloads [1], provokes the necessity of knowing the grid state best possible. Only with that knowledge, control energy as well as other control mechanisms can be used specifically.

In contrast to superordinate grid levels, hardly any measurement devices are installed in LV grids. This is because of the high costs of meters, which has to be set in context to the value of the gathered information.

Pursuant to this initial situation, the goal of this contribution is to obtain sufficient information about the grid state using as few measurement devices as possible. This way, critical grid situations (e.g. violations of power quality) shall be avoided.

Chapter II gives an overview over related references. In the following chapter III, the utilized weighted least squares (WLS) state estimation (SE) algorithm is introduced, which is adapted for usage on asymmetric LV grids. The used pseudo

978-1-5386-2344-2/17/\$31.00 (C2017 IEEE measurement values and other preliminary considerations are described in Chaper IV.

Following this, optimized meter placement and the used algorithm are introduced in chapter V. Optimization results of exemplary grids and a discussion are shown in chapter VI before chapter VII concludes the work.

\section{RELATED WORK}

As the amount of active participants in distribution grids rises, importance of SE and thus placement of measurement devices has increased.

A rule based approach along with some variable ranking was presented by Baran et al. [2]. An algorithm based on dynamic programming is presented by Muscas et al. in [3], where cost and accuracy are optimized under the constraint of getting all relevant data for protection and dispatch reasons. In [4], Singh et al. use a sequential improvement of a bivariate probability index to minimize the relative errors in voltages and angles. Based on ordinal optimization, this approach is further developed in [5]. An optimal meter placement procedure without detailed distributed generation information and under the occurrence of data loss or a bad metrological performance is described by Liu et al. in [6].

In [7], Cramer et al. describe a meter placement method using a genetic algorithm. It is based on the large number of different measurement value combinations and thus set to handle the many degrees of freedom originating from the high amount of input variables.

However, while most optimization algorithms are developed and set for distribution medium voltage grids, this contribution aims especially at low voltage grids and monitoring of arising problems in those. As a result, the importance of an asymmetric state estimation arises and each phase has to be taken into account individually when estimating the grid state. To limit the remaining optimization problem and to avoid huge computational problems, only voltage and power values as SE input are focused.

\section{ASYMMETRIC StATE ESTIMATION}

Underlying concept of the WLS SE is the maximum likelihood method, a common statistical technique used to 
solve optimization problems. Goal is to calculate the system state that is most likely according to the measured values.

Based on this method an objective function $J(x)$ is defined, which is described by the remaining error between the vector of measured values $z$ and the vector of state functions $h(x)$.

This can be formulated as

$$
J(x)=[z-h(x)]^{T} R^{-1}[z-h(x)]
$$

where $\mathrm{R}$ is the covariance matrix defining the measurement error and thus weighting the different measurement values in the least squares algorithm.

By minimizing this objective function and representing the nonlinear problem with its corresponding Taylor series, the iterative solution is found.

$$
x^{k+1}=x^{k}-\left[G\left(x^{k}\right)\right]^{-1}\left[g\left(x^{k}\right)\right]
$$

$$
\text { where } \begin{aligned}
G\left(x^{k}\right) & =\frac{\delta g\left(x^{k}\right)}{\delta x}=\left[H^{T}\left(x^{k}\right)\right] R^{-1}\left[H\left(x^{k}\right)\right], \\
g\left(x^{k}\right) & =\left[H^{T}\left(x^{k}\right)\right] R^{-1}\left[z-h\left(x^{k}\right)\right], \\
H\left(x^{k}\right) & =\frac{\delta h\left(x^{k}\right)}{\delta x}
\end{aligned}
$$

More details on the mathematical derivation can be found in [8] and [9].

The mentioned state functions $h(x)$ links the state variables $x$ to corresponding current flows and active and reactive power injections that are comparable with the measurement values. The grid is modelled with a $\pi$ equivalent circuit as described in [10], [11]. According to [12] the conductance is ignored, as the line length is smaller than 10 kilometres at a voltage level below 10 kilovolts. As described in [13], capacitive influences only have a relevant impact on the least loaded phase. As for the power quality the most critical phase is in focus, lines are considered to be decoupled.

\section{A. Asymmetric Weighted Least Squares State Estimation}

While higher grid levels can be seen as symmetrical concerning their load distribution, LV grids are mostly asymmetrical [12], [14]. This leads to more complex equivalent circuits, in which the neutral conductor impedance as well as the dependencies between the phases cannot be ignored (see also chapter IV).

Originating from this and ignoring capacitive influences as they have an insignificant role ([13], [15]), an equivalent circuit is obtained, whose electric potentials for each phase have to be considered independently as shown in Fig. 1.

Out of these potentials the state vector variables, absolute voltages and corresponding phase angles, can be calculated via nodal analysis. The state functions $h(x)$ are defined based on these variables and the equivalent circuit.

The results of [16] were taken for validating the set up functions. Furthermore, for the asymmetric state estimation calculation algorithm implemented in MATLAB, rules for assembling the Jacobian matrix $H\left(x^{k}\right)$ (consisting of derivations for each possible variable) were determined.

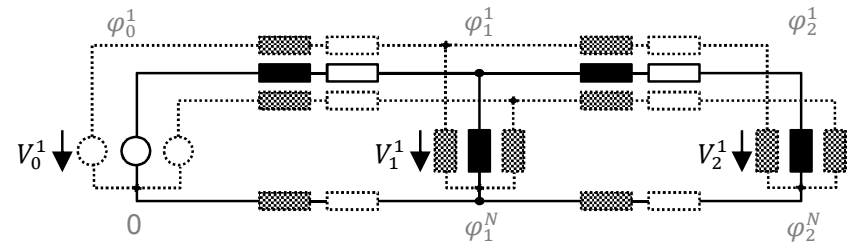

Fig. 1. Equivalent circuit of an asymmetrically loaded grid (line/dotted) and a symmetric loaded grid (line). $V_{x}^{y}$ describes the voltage at node $x$ and phase $y, \varphi_{x}^{y}$ equivalent the absolute potential.

\section{B. Real, Pseudo and Virtual Measurement Values}

As the state estimation algorithm solves complex sets of equations with six variables per grid node, it needs at least as many functions to be well defined. With the goal of achieving a sufficient SE using as little real measurement values as possible, additional input values are defined, so called pseudo and virtual measurement values [17]. This is not only necessary but can also increase the accuracy of the SE algorithm greatly.

For the simulation of placed meters, the real measurement values, only values describing a power injection, reactive as well as active, and absolute voltages are used. This is for the assumption of a bad spreading of measurement devices in LV grids, which would require meters to be added on grids in operation. The easiest way, from an economic point of view, is considered to be applying meters to customer nodes (compared to adding current measurements at branches). A time synchronization of measurements would be more complex and expensive [18]. As the absolute phase angle of a certain LV grid node is not important for the typical customer angles are not taken into account at this point. Real measurements are seen as reference values and thus their trust level, the weighting by the covariance matrix $\mathrm{R}$, is defined as 1 .

Pseudo measurement values are, in accordance with the real values, only active and reactive power injections and absolute voltages. They are freely definable and therefore the trust level is set to 0.1 for power values and to 0.001 for voltages $(10 \% / 0.1 \%$ of the real values $)$. This difference is explainable by the different effect of voltage and power values on the SE results as they have a relation via the line impedance. For the described tests in this paper the power values are set to 0 , as it has the effect of maintaining the SE results in realistic dimensions without having additional information of the node. For the absolute voltages, a linear interpolation of known neighbouring voltages being defined by real measured values has been carried out (3).

$$
U_{i}=\frac{1}{|N|} \sum_{j \in N} U_{j}
$$

where $N$ is the set of all nodes connected to node $i$.

The third set of input parameters is derivated from structural information of the grid. In particular, the fed in power at junction nodes is set to 0 with a trust level of 10 , ten times higher than measured values. This does not prevent the SE from assuming small amounts of power being injected, but as it is only up to $1 \%$ of real power values the results are scarcely influenced. 


\section{DATa SOURCE AND PRELIMINARY CONSIDERATIONS}

For testing the optimization method, four different real low voltage grids as well as modified versions of those have been implemented (Table I). Load data has been calculated according to realistic scenarios in [19] (grids rl-r4) respectively freely set in order to create certain load situations (grids $m 1-m 4$ ). The used measurement values as inputs for the SE are subsets of those datasets. Furthermore, the values serve as reference points for the calculation of estimation errors and subsequent benchmarking of results.

The studies described and presented in chapter V and VI have been carried out for all grids described in Table I. The different load situations are as follows:

- Homogeneous: Grid load is either positive or negative at all nodes.

- Inhomogeneous: Grid areas (separated at node 3, Fig. 2) have different load flow directions. One half is consuming power, the other one is producing.

- Asymmetrical: Total node loads are split up asymmetrical on the three phases.

\section{A. Exemplary Grid Setup}

Figure 2 shows the general setup of grids $m 1-m 3$ and $r 1$. Numbers 1 to 17 mark the grid nodes that were taken into account for the SE, whereby node 1 is the slack node being considered ideally connected to a busbar, node 9 . Node 17 (dotted) has been added to the modified grids in order to create a worse load situation - it does not exist in grid $r 1$. The line lengths and directions shown in Figure 2 are a geographic representation.

\section{B. Influence of Measurement Errors}

Real measurement values in general contain errors, not least because of temporal discrepancies between different measurements. As this error spreads to the SE quality, the SE algorithm is tested with measurement values superimposed by a normal distributed measurement error of maximum $1 \%$ (4). Statistical outliers occurring because of the normal distribution were excluded for the assumption of an implemented bad data detection [20] as to improve the comparability of the results.

TABLE I

REAL AND MODIFIED TEST GRIDS USED FOR THE OPTIMAL METER PLACEMENT

\begin{tabular}{|c|c|c|c|c|c|}
\hline \multirow{2}{*}{ Grid } & Nodes & $\begin{array}{c}\text { Load } \\
\text { (kVA) }\end{array}$ & $\begin{array}{c}\text { Prod. } \\
\text { (kVA) }\end{array}$ & $\begin{array}{c}\text { Voltage } \\
\text { Range (V) }\end{array}$ & Load Situation \\
\hline $\mathbf{r} 1$ & 16 & 8.8 & 45.2 & $236.9-238.7$ & Homogeneous \\
\cline { 2 - 6 } $\mathbf{r 2}$ & 30 & 168.0 & 33.2 & $213.4-237.5$ & Asymmetrical \\
\cline { 2 - 6 } $\mathbf{r 3}$ & 85 & 127.9 & 7.6 & $217.7-226.8$ & Homogeneous \\
\cline { 2 - 6 } $\mathbf{m} \mathbf{m}$ & 148 & 105.7 & 32.4 & $224.1-230.1$ & Homogeneous \\
\cline { 2 - 6 } $\mathbf{m} 2$ & 17 & 82.5 & 84.9 & $222.1-246.7$ & Inhomogeneous \\
\cline { 2 - 6 } $\mathbf{m 3}$ & 17 & 14.0 & 220.0 & $230.5-253.0$ & Homogeneous \\
\cline { 2 - 6 } $\mathbf{m 4}$ & 85 & 311.9 & 8.9 & $206.2-236.7$ & Homogeneous \\
\cline { 2 - 6 } & 17 & 86.8 & 72.2 & $221.0-255.7$ & Asymmetrical \\
\hline
\end{tabular}

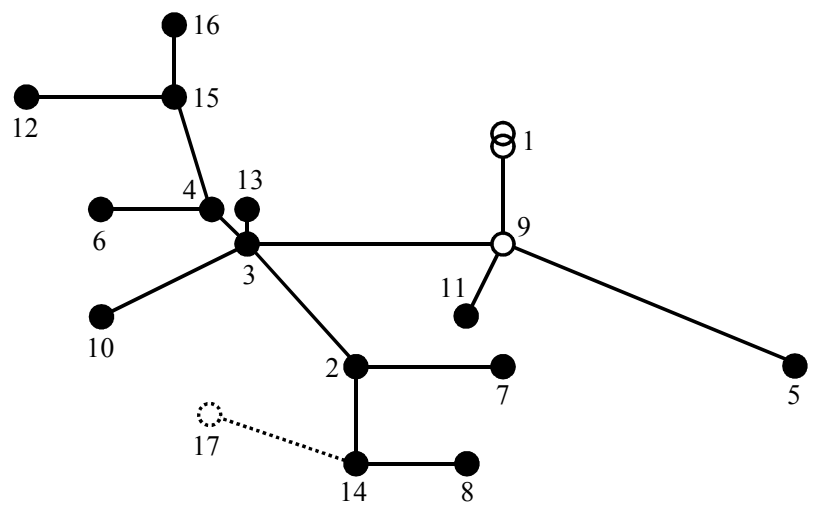

Fig. 2: Graphical representation of grid $r l$ (without dotted node 17) and grids $m 1-m 3$

SE error (\%) Meter positions: I node 16 I node 1, 3, 16, 17 I all nodes

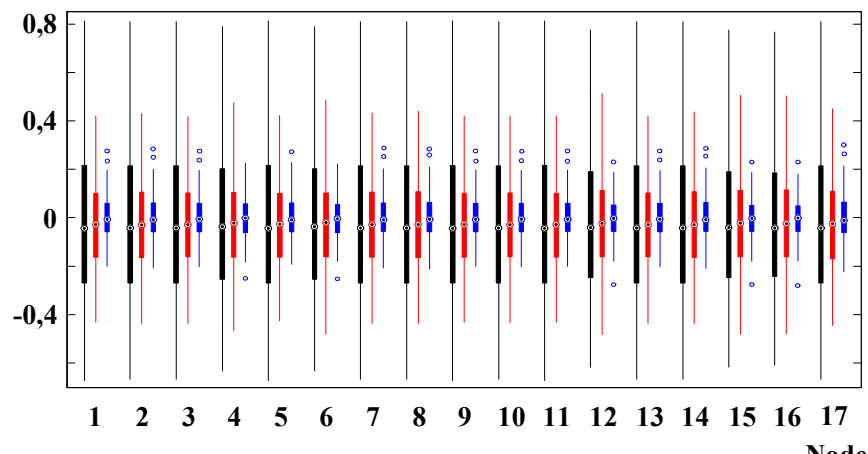

Node

Fig. 3: Estimation Error of 200 SE with normal distributed measurement error, subject to node number and different meter distributions.

$$
e_{\max }=1 \%, 3 \sigma=1 \% \text { deviation }
$$

Fig. 3 shows the estimation errors on each single grid node subject to different meter distributions in grid $m 1$, which is loaded inhomogeneously. For the distributions, 200 SEs were calculated in order to minimize statistical errors. Each grid node has three assigned bars, which show the results for 1 meter $\left(1^{\text {st }}\right), 4$ meters $\left(2^{\text {nd }}\right.$ bar) and 17 meters $\left(3^{\text {rd }}\right.$ bar) placed. The boxplot quantiles are set to 0.25 and 0.75 . The results plotted in Fig. 3 show that the remaining estimation errors are smaller than the causing measurement error, even for the worst possible situation of only 1 meter installed. This leads to neglecting measurement errors in the further studies, as it is transmitted to the SE directly. Considering the limited influence and the worse comparability all simulations were run with exact values.

\section{Relevance of Asymmetric Approach}

As specified before, an asymmetric state estimation approach is used for the calculations of exemplary low voltage grids. In order to compare the performance of a normal SE and the asymmetric one grid $m 3$ has been modified to be asymmetrically loaded - and thus causing a current on the neutral conductor. The asymmetry was concentrated on node 16 , injecting a power of $3.5 \mathrm{kVA}$ on one phase while loading the two other phases. 


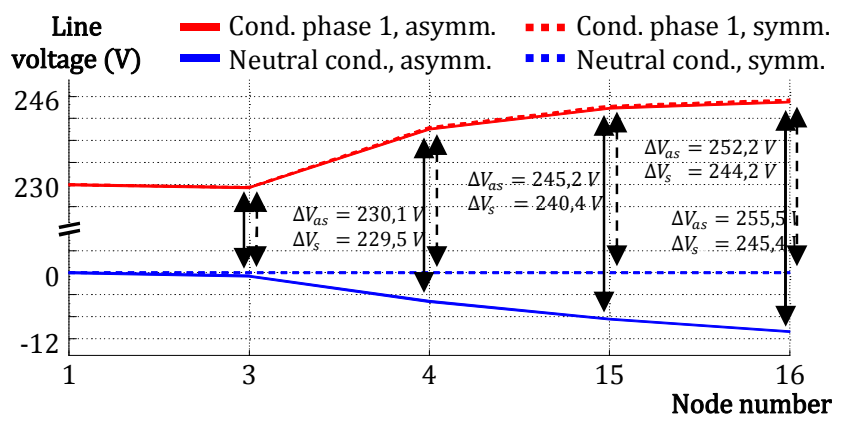

Fig. 4: Comparison of potentials along an asymmetrically loaded grid branch, asymmetric vs. symmetric SE approach.

The results of both SE approaches can be seen in Fig. 4. Blue and red lines represent the absolute voltage values along the lines between each two nodes (x-axis). Solid lines show results of the asymmetrical SE while dashed lines represent the symmetrical SE.

The results shown point out that using a symmetric approach SE algorithm, the currents on the neutral conductor cannot be taken into account and thus the voltage drop along the cable is calculated wrongly. This leads to inaccurate results (in this case about $5 \%$ deviation). As shown here, such an error could evoke a misjudgement of the system state, as the violation of a voltage band is not detected.

Comparing calculation times of both approaches, the asymmetric SE needs about 30 times longer for the calculations carried out than the normal SE. Nevertheless, in this contribution the asymmetric SE is seen as beneficial, as the calculation time is still feasible.

While an alternative to the asymmetric SE would be to gather additional measurement values of neutral conductors, this would not be practically applicable due to higher hardware investment cost.

\section{OPTIMIZED METER PlacEMENT}

With the described WLS SE algorithm, the required amount of meters and the positioning of meters is analysed. Thus, a good cost-benefit ratio monitoring low voltage grids shall be achieved. As all power flow values are strictly related to the node voltages, which are used as state vector variables, absolute voltages form the quality criteria. The errors are therefore defined as the relation of the resulting SE state variables and the known real voltage levels at each individual node (5).

$$
e_{i, \text { res }, S E}=\left(\frac{\left|U_{i, S E}\right|}{\left|U_{i, \text { real }}\right|}-1\right) \cdot 100 \%
$$

Determining estimation errors for any linear combination of placed meters is not applicable for larger grids - the number of possible combinations scales with a factor of 2 for every node. As the distribution of meters and thus the estimation error is strongly dependent on grid and load setups, a method to investigate grids individually in reasonable time has to be developed. Originating from this

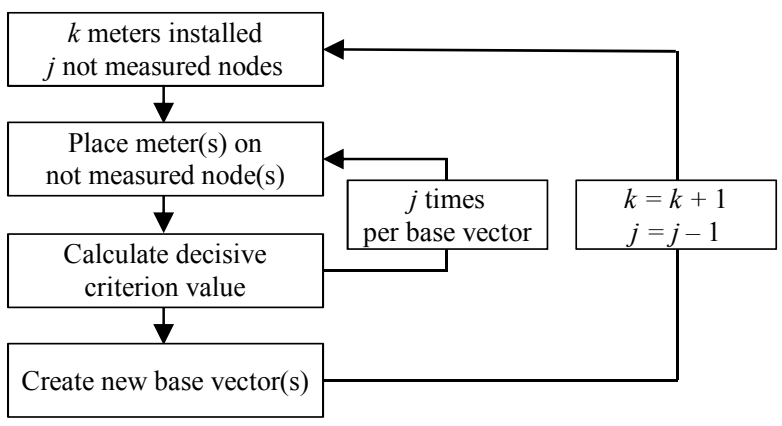

Fig. 5: Flow chart of meter placement search algorithms.

idea, genetic optimization algorithms were implemented in order to achieve optimized results within acceptable computational time.

For the tests base vectors were used as identifier whether a certain nodes is measured (logical 1 ) or not (logical 0 ). These are further developed in each iteration of the SE and thus also represent the results of the genetic algorithm.

Figure 5 shows the basic procedure for the following optimization methods:

- Method 1: Prioritisation of the least influenceable node

In each iteration step, one additional meter is placed in the grid, successively at every non-measured node in the grid. The resulting estimation errors of each run are added up for each node individually, forming the decisive criterion $c$ (6). The new meter for the next iteration step is placed at the node that has the biggest error and thus is least affected by other nodes.

For each node $n: \quad c=\sum_{S E \text { runs }} e_{n, \text { res,SE }}$

- Method 2: Prioritisation of best result(s) with minimal sum of quadratic estimation errors

While the principal procedure is the same as before, the decisive criterion is the sum of quadratic weighted estimation errors of all nodes (7). The meter whose placement leads to a minimal sum is chosen as the best result. For the next iteration step, the best results (amount is definable) are taken as new base vectors from which the search for the optimal placement of one more meter starts.

For each run $j: \quad c=\sum_{\text {nodes }} e_{j, \text { res }, S E}$

- Method 3: Prioritisation of best result(s) with minimal sum of quadratic estimation errors, search depth of 2

Following the same basic idea as in Method 2, not only one additional meter per run is placed but the distribution of two additional meters in each possible combination is investigated. While the results of placing two more measuring devices are compared, the next iteration base vectors are created using the meter positions with the best results separately (assuming meters at positions $\mathrm{X}$ and $\mathrm{Y}$ are best, the new base vectors are created by adding a meter to $\mathrm{X}$ respectively $\mathrm{Y}$ separately).

The computing times as well as the results of those three methods differ, as especially the amount of SE runs increases 
with a growing complexity of the algorithm. Though it is possible that a local minimum instead of an optimal distribution is found, investigations showed that Method 2, following the five best base vectors, provides the best results in relation to the time needed by the algorithm - and thus is chosen for all further investigations discussed in this paper. As the absolute time hinges with the processing capacity, times were compared using the same CPU for all runs.

\section{RESUlTS AND DISCUSSION}

Using the genetic algorithm, the described grids in Table 1 were analysed and the results compared with respect to the general amount of required meters and best possible meter positioning. Each installed meter is considered to measure voltage and power values on all three phases. As decisive criterion the mean error as well as the quadratic mean error of all estimated absolute voltages of grid nodes are compared.

\section{A. Amount of measurement devices installed}

The results of the optimized meter placement algorithm are exemplarily shown for grid $m 1$ in Fig. 6. In order to increase the informative value, not only the errors for the best meter placement but also for the $4^{\text {th }}$ and $7^{\text {th }}$ best meter distributions are shown. The discussion focuses on the quadratic error, the mean error is given as additional information about the range of variation.

The inhomogeneously loaded grid was set up to have one region that shows a quite big load while the other region injects a lot of power - in order to create a critical grid situation. In this example, it leads to a maximum voltage drop of $24.7 \mathrm{~V}$ between two nodes. This voltage drop causes a high error of $5.5 \%$ when only one node is measured, caused by the SEs voltage interpolation.

Considering the remaining errors for 2 and more meters, it can be seen that there is a rapid improvement adding only a few more measurement devices in the grid. Installed at optimized positions, the voltage interpolation leads to significantly better results - the $2^{\text {nd }}$ installed meter helps to differ load zones while a $3^{\text {rd }}$ helps to fix the 'common' node between those zones.

As those results only apply for this specific grid setup, the corresponding results of all test grids are compared against each other in Fig. 7.

This again shows the strong dependency of the remaining error and the individual grid setup and load situation. The more even the load is spread and the more homogeneous the general grid setup is, the better the results. The greater asymmetries and the more disproportionate the node loads are, the bigger the error.

A noticeable finding analysing Fig. 7 are the good SE results with only a few measured nodes. This applies for large grids with up to 148 nodes as well as for small ones. Investigations of the bar graphs for more than 6 installed meters showed that the curves tend asymptotically against zero. In all grids, no greater improvements per additional meter for more than 5 meters were noticeable.

\section{Estimation error (\%)}

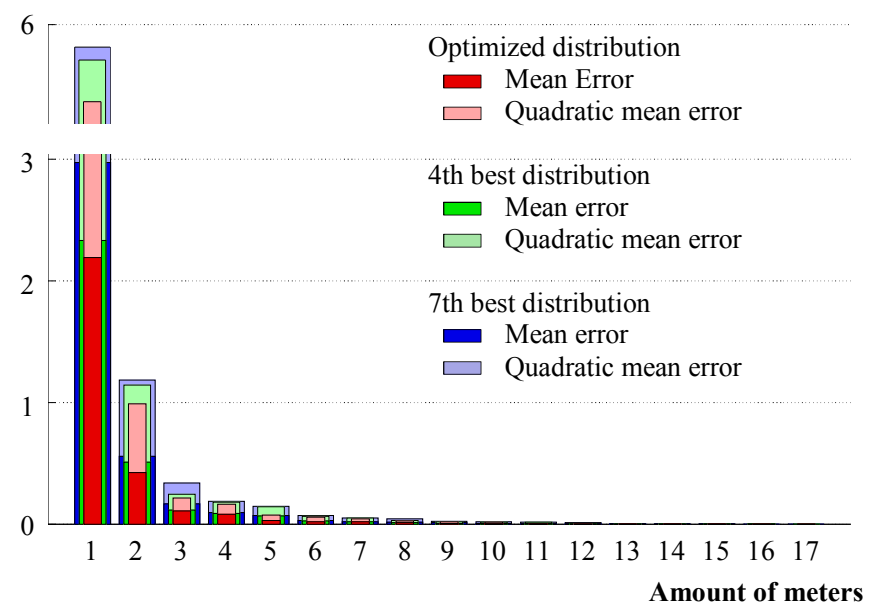

Fig. 6: Estimation error depending on the amount of installed meters and meter distribution, grid $m 1$

\section{Estimation error (\%)}

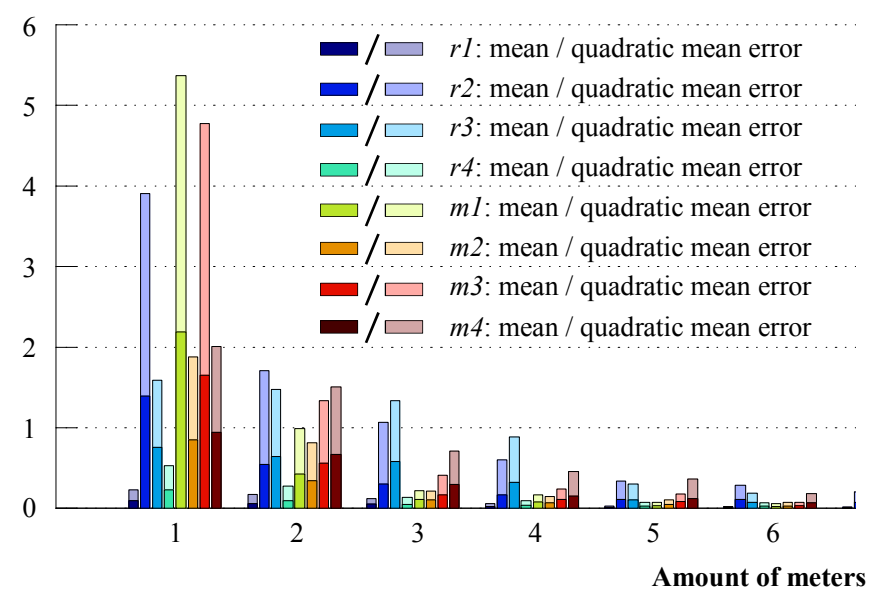

Fig. 7: Comparison of estimation errors depending on the amount of installed meters of all grids

This is explainable by grid characteristics. More nodes in a grid do not automatically lead to a higher amount of clustered grid areas, which can be estimated with an acceptable error by a single meter. In addition, asymmetrical load flows are increasingly evened out.

\section{B. Identification of significant measurement points}

After having the focus on the overall estimation error, now the exact meter positions are analysed. Again, as grid situations are very specific, the results of different grids are analysed in order to identify certain grid nodes in relation to general physical as well as load specific situations.

The optimized meter placement for grid $m 1$ is shown in Fig. 8, where the grey boxes indicate the nodes where the measurement devices are placed. Crosses represent the estimation error at each individual node; green indicates an improvement compared to the result with one meter less, red is a deterioration. The y-axis is scaled the same for all graphs in order to increase comparability. 


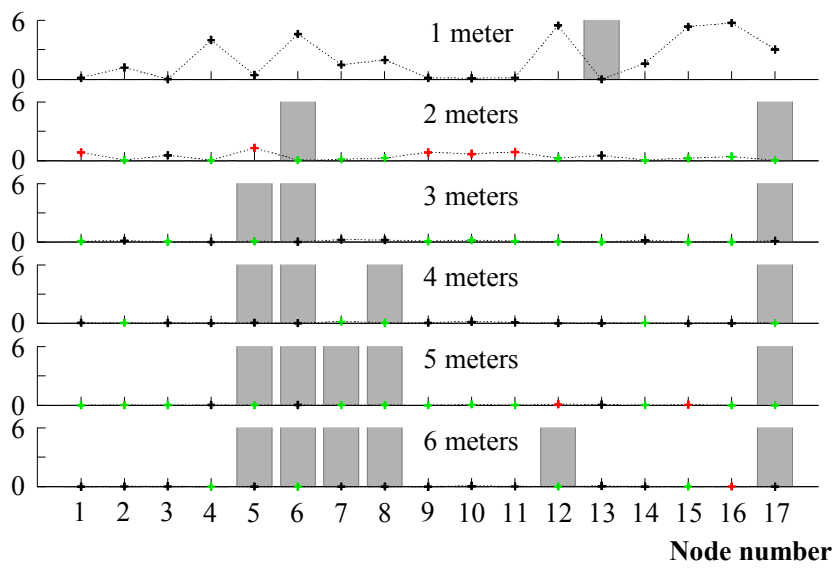

Fig. 8: Optimal meter placement for 1 to 6 meters, grid $m 1$. Grey boxes indicate the meter positions.

Analysing Fig. 8 considering Fig. 2, the inhomogeneous load situation gets visible. Only one meter would be best placed at a more or less neutral point in the grid, a node that represents the voltage that is closest to the mean grid voltage. The significance of the SEs resulting grid state of course is very low.

The distribution of two meters already leads to a relatively good cost-benefit ratio, placing them at high load nodes in contrary areas of the grid and thus capturing the special load situation. The results are furthermore improved by placing a third meter at node 5 , which is rather close to the transformer and the other nodes with a high deviation. Thus the voltages in this 'third area' of the grid are interpolated more precisely and therefore a better state estimation is achieved.

\section{CONCLUSIONS}

In this paper, optimized meter placement in LV grids was investigated. The utilized asymmetric WLS SE was introduced and the importance of the asymmetric approach highlighted. Measurement errors were excluded for further simulations as comparability of results would decrease. The SE input parameters, real, pseudo and virtual measurement values, and their weighting were discussed. Out of three introduced methods for meter placement, a genetic algorithm using the quadratic estimation error as quality criterion was chosen. Subsequent studies on exemplary real grids showed that, weighting monitoring costs against the benefit of more meters installed, even for big grids a small amount of meters is sufficient in order to achieve good estimation results. In all grids, five respectively three meters led to satisfying resulting estimation errors. The remaining quadratic errors with meters placed optimized were lower than $0.4 \%$ of grid voltage (less than 1 Volt), what is considered reasonable in terms of monitoring LV grids. Focusing on the placement of measurement devices, simulations showed that metering of node clusters respectively grid areas showing a certain load situation is most important.
[1] D. A. Haughton and G. T. Heydt, "A Linear State Estimation Formulation for Smart Distribution Systems," in IEEE Transactions on Power Systems, vol. 28, no. 2, pp. 1187-1195, May 2013.

[2] M. E. Baran, Jinxiang Zhu and A. W. Kelley, "Meter placement for real-time monitoring of distribution feeders," in IEEE Transactions on Power Systems, vol. 11, no. 1, pp. 332-337, Feb. 1996.

[3] C. Muscas, F. Pilo, G. Pisano and S. Sulis, "Optimal Allocation of Multichannel Measurement Devices for Distribution State Estimation," in IEEE Transactions on Instrumentation and Measurement, vol. 58, no. 6, pp. 1929-1937, June 2009.

[4] R. Singh, B. C. Pal and R. B. Vinter, "Measurement Placement in Distribution System State Estimation," in IEEE Transactions on Power Systems, vol. 24, no. 2, pp. 668-675, May 2009.

[5] R. Singh, B. C. Pal, R. A. Jabr and R. B. Vinter, "Meter Placement for Distribution System State Estimation: An Ordinal Optimization Approach," in IEEE Transactions on Power Systems, vol. 26, no. 4, pp. 2328-2335, Nov. 2011.

[6] J. Liu, F. Ponci, A. Monti, C. Muscas, P. A. Pegoraro and S. Sulis, "Optimal Meter Placement for Robust Measurement Systems in Active Distribution Grids," in IEEE Transactions on Instrumentation and Measurement, vol. 63, no. 5, pp. 1096-1105, May 2014.

[7] M. Cramer, P. Goergens, F. Potratz and A. Schnettler, "Genetic Algorithm for Optimal Meter Placement and Selection in Distribution Grid State Estimation," International ETG Congress 2015; Die Energiewende - Blueprints for the new energy age, Bonn, Germany, 2015, pp. 1-7.

[8] K. D. Jones, "Three-phase linear state estimation with phasor measurements," master's thesis, Virginia Polytechnic Institute and State University, May 2011.

[9] A. Abur and A. G. Expósito, Power system state estimation: theory and implementation. New York/Basel: Marcel Dekker, Inc., 2004.

[10] V. Crastan, Elektrische Energieversorgung 1: Netzelemente, Modellierung, stationäres Verhalten, Bemessung, Schalt- und Schutztechnik, ed., vol. III. Berlin/Heidelberg: Springer Verlag, 2012.

[11] M. Bendrat: "Analyse der Spannungsqualität in Verteilnetzen auf der Niederspannungsebene sowie Realisierung geeigneter Kompensationsmaßnahmen," Ph.D. dissertation, Fern-Universität in Hagen, 2010.

[12] K. Heuck, K.-D. Dettmann and D. Schulz, Elektrische Energieversorugng: Erzeugung, Übertragung und Verteilung elektrischer Energie für Studium und Praxis, ed., vol. IX. Wiesbaden: Springer Vieweg Verlag, 2013.

[13] W. H. Kersting and W. H. Phillips, "Distribution feeder line models," in IEEE Transactions on Industry Applications, vol. 31, no. 4, pp. 715-720, Jul./Aug. 1995.

[14] J. C. M. Vieira, W. Freitas and A. Morelato, "Phase-decoupled method for three-phase power-flow analysis of unbalanced distribution systems," in IEE Proceedings - Generation, Transmission and Distribution, vol. 151, no. 5, pp. 568-574, 13 Sept. 2004.

[15] N. Neusel-Lange, "Dezentrale Zustandsüberwachung für intelligente Niederspannungsnetze," Ph.D. dissertation, University of Wuppertal, Oct. 2013.

[16] C. N. Lu, J. H. Teng and W. H. E. Liu, "Distribution system state estimation," in IEEE Transactions on Power Systems, vol. 10, no. 1, pp. 229-240, Feb. 1995.

[17] L. Holten, A. Gjelsvik, S. Aam, F. F. Wu and W. H. E. Liu, "Comparison of different methods for state estimation," in IEEE Transactions on Power Systems, vol. 3, no. 4, pp. 1798-1806, Nov. 1988.

[18] S. Bolognani, R. Carli and M. Todescato, "State estimation in power distribution networks with poorly synchronized measurements," $53 \mathrm{rd}$ IEEE Conference on Decision and Control, Los Angeles, CA, 2014, pp. 2579-2584.

[19] M. Uhrig, N. Meyer-Huebner and T. Leibfried, "Approach for determining the demand for stationary energy storages in low voltage distribution grids," XIII Spanish-Portuguese conference on electrical engineering, Valencia, Spain, 2013.

[20] D. Waeresch, R. Brandalik, W. H. Wellssow, J. Jordan, R. Bischler and N. Schneider, "Bad data processing for low voltage state estimation systems based on smart meter data," CIRED Workshop 2016, Helsinki, 2016, pp. 1-4. 